\title{
Detecting DNA via the inflammasome
}

Endosomal Toll-like receptor 9 (TLR9) and the cytoplasmic sensor DAI (also known as ZBP1) have previously been identified as intracellular receptors for DNA that trigger a type I interferon (IFN) response. Now, Muruve et al. report that activation of the inflammasome, a multi-protein complex involved in the innate immune response against many pathogens, is also important for the generation of an effective inflam-

...the

inflammasome

response operates

independently

of other known

DNA-sensing mechanisms... extracellular adenoviral DNA, indicating that neither virion internalization alone nor extracellular DNA could trigger the inflammasome.

A closer examination of inflammasome components revealed that the cytoplasmic receptor NALP3 (NATCHT-, leucine-rich-repeat- and pyrin-domain-containing protein 3; also known as NLRP3 or cryopyrin) and its adaptor, ASC (apoptosisassociated speck-like protein containing a CARD), are essential for sensing viral DNA, as macrophages from mice deficient in either of these proteins had significantly reduced inflammasome activation in response to adenoviruses. NALP3 and ASC are thought to function in the recruitment of caspase- 1 to the inflammasome complex, which thereby leads to the maturation of pro-IL-1 $\beta$. An established model of adenovirus infection in mice was used to demonstrate that NALP3, ASC and caspase- 1 were all required for a maximal immune response to DNA viruses in vivo. As evidence that the inflammasome response operates independently of other known DNAsensing mechanisms, the authors showed that, following exposure to adenovirus DNA, caspase-1 processing was intact in macrophages that lack both TLR9 and the TLR adaptor protein MyD88. Therefore, inflammasome activation is required in vitro and in vivo for mounting effective immune responses against DNA viruses.

Interestingly, the presence of non-viral DNA in the cytoplasm (of Escherichia coli, mammalian cells or synthetically derived) also initiated inflammasome activation in THP-1 cells and mouse macrophages. Such a response was not elicited by mammalian RNA, polyinosinic-polycytidylic acid or RNA viruses, demonstrating that inflammasome activation is specifically triggered by DNA.

Despite the importance of both the type I IFN response and the inflammasome in antiviral immunity, the authors found that inflammasome activation triggered by DNA sensing occurred independently of IFN production. Caspase- 1 and pro-IL-1 $\beta$ maturation in response to cytoplasmic DNA occurred nearly as efficiently in cells deficient in the type I IFN receptor. Conversely, signalling by IFN-regulatory factor 3 (which is triggered early in the type I IFN pathway) following infection with adenovirus was functional in NALP3- or ASCdeficient macrophages. Therefore, the inflammasome pathway is distinct and independent from the type I IFN response that is triggered following DNA recognition in the cytoplasm.

So, in addition to identifying a new pathway for host and microbial DNA recognition, this study might provide clues to the pathology of autoimmune diseases such as systemic lupus erythematosus and chronic arthritis, which are often associated with autoreactive antibodies specific for self DNA and increased levels of IL-1 $\beta$. That such conditions might involve aberrant inflammasome function is a possibility that warrants further investigation.

Sarah Allan

ORIGINAL RESEARCH PAPER Muruve, D. A. et al. The inflammasome recognizes cytosolic microbial and host DNA and triggers an innate immune response. Nature 452, 103-107 (2008). 